$11-1995$

\title{
An Algorithmic Approach to Loop Shaping With Applications to Self-Tuning Control Systems
}

Zhiqiang Gao

Cleveland State University, Z.GAO@csuohio.edu

Follow this and additional works at: https://engagedscholarship.csuohio.edu/enece_facpub

Part of the Controls and Control Theory Commons

How does access to this work benefit you? Let us know!

\section{Publisher's Statement}

NOTICE: this is the author's version of a work that was accepted for publication in Journal of the Franklin Institute-Engineering and Applied Mathematics. Changes resulting from the publishing process, such as peer review, editing, corrections, structural formatting, and other quality control mechanisms may not be reflected in this document. Changes may have been made to this work since it was submitted for publication. A definitive version was subsequently published in Journal of the Franklin Institute-Engineering and Applied Mathematics, 332, 6, (11-01-1995); 10.1016/0016-0032(95)00053-4

\section{Original Citation}

Zhiqiang, G. (1995). An Algorithmic Approach to Loop Shaping with Applications to Self-tuning Control Systems. Journal of The Franklin Institute, 332(6), 643-656.

\section{Repository Citation}

Gao, Zhiqiang, "An Algorithmic Approach to Loop Shaping With Applications to Self-Tuning Control Systems" (1995). Electrical Engineering \& Computer Science Faculty Publications. 70.

https://engagedscholarship.csuohio.edu/enece_facpub/70

This Article is brought to you for free and open access by the Electrical Engineering \& Computer Science Department at EngagedScholarship@CSU. It has been accepted for inclusion in Electrical Engineering \& Computer Science Faculty Publications by an authorized administrator of EngagedScholarship@CSU. For more information, please contact library.es@csuohio.edu. 


\section{An Algorithmic Approach to Loop Shaping with Applications to Self-tuning Control Systems}

by ZHIQIANG GAO

Department of Electrical Engineering, Cleveland State University, Cleveland, OH 44115 , U.S.A.

ABSTRACT: An algorithmic approach to feedback control design is introduced. It simplifies the existing iterative design process, which is often tedious, by reducing the design problem to solving a set of linear algebraic equations. The algorithmic nature of such an approach makes it attractive to not only off-line designs but also self-tuning control systems, where the compensators are continuously tuned on-line as the dynamics of the physical process vary with time. This is demonstrated in the example where the proposed algorithm is implemented for an industrial tension regulation system with successful simulation results. Extensions of the algorithm to multi-input and multi-output systems, as well as discrete time systems, are also introduced.

\section{Introduction}

It is well known in control theory that much information concerning performance, stability and robustness of a linear time-invariant control system can be obtained from its loop gain frequency response. This insightful relationship leads to the principle of loop-shaping design techniques; see, for example, (1-3). In loopshaping design, the closed-loop specifications are translated to the constraints on the loop gain transfer function, which can be met by an appropriate choice of the compensator. The combination of Nyquist and the Bode plots, as well as Nichol's charts, provided powerful graphical tools for single-input and single-output (SISO) control system design. Design techniques based on loop frequency response were also developed for multi-input and multi-output (MIMO) systems with variations.

The use of loop-shaping techniques requires skill and experience. Although there are guidelines on how to select the compensator to manipulate the loop gain frequency response, the design is carried out in a largely cut-and-try procedure, which can be tedious at times. A new design method is proposed in this paper as an alternative to the iterative design approach. It reduces the design problem to solving a set of linear algebraic equations which can be carried out by a computer.

The new design process not only reduces the burden on the designer but also makes it possible for a more autonomous design that can be implemented on-line. Note that the previous loop-shaping techniques are mainly off-line design methods. The designer selects the compensator based on the observations of the Nyquist and Bode plots of the plants and the desired loop gain. Such interactions are not 


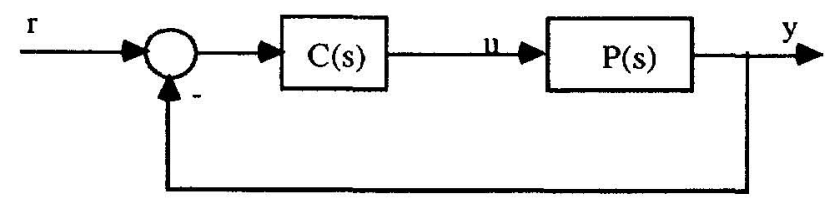

Fig. 1. A feedback control system.

necessary in the proposed approach, where a computer algorithm determines the appropriate compensator from the frequency response of the plant and the loop gain constraints. The simplicity and the algorithmic nature of such an approach make it attractive to self-tuning control systems where the compensators are continuously tuned on-line as the dynamics of the physical process vary with time.

The automatic on-line tuning of controllers poses many challenges to control engineers. The need to tune controllers arises from practical control problems. Most physical systems are, in general, nonlinear and time-varying systems. Yet the majority of the current control techniques are based on linear and time-invariant models. These models are usually obtained from the approximation of the plants in the neighborhood of an operating point. In many control applications, however, the system dynamics could change significantly from one operating point to the other, and the controllers must be adjusted, or tuned, in order to maintain the performance and stability. Due to the complexity of the problem, main research activities on self-tuning controllers have so far been largely restricted to controllers of simple structure, such as PID controllers; see, for example, (4). With the proposed algorithm, it is now possible to perform auto-tuning for a large class of controllers.

The new design method is developed based on the matrix interpolation theory (5) where the loop gain constraints are expressed as interpolation constraints. The design problem is formulated as a polynomial matrix interpolation problem and is described in Section II. A novel self-tuning control system and its implementation in an industrial tension control system is shown in Section III. The extension of the proposed approach to discrete time systems and MIMO systems are described in Section IV. Finally, some concluding remarks are included in Section V.

\section{A New Design Technique}

Consider the linear, time-invariant, SISO control system in Fig. 1. $P(s)$ is the transfer function of the plant, $C(s)$ is the compensator to be determined. A classical approach to feedback design is to work directly with the loop gain transfer function, $L(s)=P(s) C(s)$. The stability and performance specifications are interpreted as constraints on the loop gain frequency response, $L(j \omega)=P(j \omega) C(j \omega)$. From these constraints, the designer knows that the $|L(j \omega)|$ should be large up to a frequency and small beyond another frequency; the designer also knows roughly where the crossover frequency, $\omega_{c}$, should be from the transient response requirements. The Nyquist stability theorem puts additional constraints on $L(j \omega)$ in terms of the encirclement of the $-1+j 0$ point by the graph of $L(j \omega)$, the gain and phase margins, etc. 
In the existing loop-shaping design techniques, $C(s)$ is determined iteratively, such that $L(j \omega)$ satisfies all constraints; see for example, (1). This process of finding the appropriate $C(s)$ requires a great deal of human intuition and experience. Compromises are often made in the transient response specifications, the compensator complexity, the actuation limit and the stability robustness, etc. In the following, a new procedure is introduced.

\subsection{Problem formulation}

Let the design specifications be expressed as interpolation constraints of the form:

$$
L\left(j \omega_{i}\right)=\alpha_{i}, \quad i=1, l,
$$

where $\alpha_{i}$ are complex numbers. Note that this is a reasonable assumption since most design specifications, such as a command following in certain frequency range, crossover frequency, gain and phase margins, stability robustness against high frequency unmodeled dynamics, etc., can be translated as constraints on magnitude and phase of $L(j \omega)$ at a set of frequencies, $\left\{\omega_{i}\right\}$. Then, the design problem becomes to find a compensator $C(s)$ such that:

$$
L\left(j \omega_{i}\right)=P\left(j \omega_{i}\right) C\left(j \omega_{i}\right), \quad i=1, l,
$$

where $P\left(j \omega_{i}\right)$ is the given frequency response of the plant evaluated at $\omega_{i}$. Writing $L\left(j \omega_{i}\right)=\alpha_{i}$ and $P\left(j \omega_{i}\right)=\beta_{i}, i=1, l$, the numerator and denominator coefficients of $C(s)$ can be obtained by solving the set of linear algebraic equations:

$$
C\left(j \omega_{i}\right) \beta_{i}=\alpha_{i}, \quad i=1, l .
$$

Given the degree of $C(s), \alpha_{i}$ and $\beta_{i}$, solving $C(s)$ from Eq. (2.3) can be seen as the rational function interpolation problem. This problem is also known as the transfer function curve-fitting problem (6). It is similar to the system identification problem, where the transfer function is to be determined from the frequency response of the plant (7-9).

\subsection{Solving the interpolation problem}

Many control system constraints and properties can be expressed as interpolation constraints of transfer functions or transfer function matrices. For example, the curve-fitting problem discussed above is a special case of the matrix interpolation problem. The recent development in matrix interpolation theory (5) offers a new theoretical framework in which various algebraic aspects of the matrix interpolation problems are explored. Computer algorithms $(\mathbf{8}, \mathbf{9})$ are developed to solve practical problems. It is shown, in the following, that the design problems formulated in Eqs (2.1)-(2.3) can be effectively solved using the matrix interpolation theory and algorithms.

Let $C(s)$ be $C(s)=n(s) / d(s)$, where $n(s)$ and $d(s)$ are numerator and denominator polynomials, respectively. The problem in Eq. (2.3) is equivalent to determining the polynomial matrix $[n(s),-d(s)]$ which satisfies 


$$
\left[n\left(j \omega_{i}\right),-d\left(j \omega_{i}\right)\right]\left[\begin{array}{l}
\beta_{i} \\
\alpha_{i}
\end{array}\right]=0, \quad i=1, l,
$$

where $l$ is the number of constraints.

Given column degrees of $n(s)$ and $d(s), d_{1}$ and $d_{2}$, and $l$ constraints $\left\{j \omega_{i}, \beta_{i}, \alpha_{i}\right\}$, the matrix $[n(s),-d(s)]$ can be uniquely determined from (5):

$$
[N,-D]\left[S_{l}, C\right]=[0, E],
$$

where

$$
[n(s),-d(s)]=[N,-D] S(s)
$$

with

$$
\begin{gathered}
S(s)=\operatorname{blk} \operatorname{diag}\left\{\left[1, s, \ldots, s^{\left.d_{1}\right]^{\prime}}\right\}, \quad i=1,2\right. \\
S_{l}:=\left[S\left(j \omega_{1}\right) c_{1}, \ldots, S\left(j \omega_{l}\right) c_{i}\right] .
\end{gathered}
$$

Here $N$ and $D$ are row vectors which contain the coefficients of $n(s)$ and $d(s)$, respectively; $c_{i}=\left[\beta_{i}, \alpha_{i}\right]^{\prime}$. Equations $[N, D] C=E$ represent $k$ additional constraints on the coefficients; $k$ is the number of columns of $C$ or $E$ and it is taken to be $k=\left(\Sigma d_{i}+(p+m)\right)-l$. Furthermore, $C$ is selected so that $\left[S_{l}, C\right]$ has full rank, assuming $S_{i}$ has full rank (5); in this way a unique solution exists for any $E$. These additional conditions can be used, for example, to ensure the properness of $C(s)$, or to make $d(s)$ a monic polynomial. Detailed algebraic properties of this fitting problem are discussed in $(5,7)$.

Note that Eq. (2.5) can be solved as a weighted least-square problem (10) and frequency weighting can be easily implemented to reflect the degree of importance of each loop gain constraint. The solutions can be obtained in one step by solving Eq. (2.5), which is a set of linear algebraic equations.

2.2.1. Stability. Since stability is one of the most important characteristics of any feedback system, it should take the highest priority in design. In selecting loop gain constraints, the gain and phase margin should be large enough to allow inaccuracies from approximations in both modeling and design process. Frequency weighting can be used to make the stability constraints dominant in the cost function.

2.2.2. The order of the compensator. One can always find a $C(s)$ that satisfies all constraints in Eq. (2.3) by picking the degree of $C(s)$ high enough. In practice, however, it is often required to find the compensator of lowest order which meets all the specifications. Furthermore, the loop gain constraints are usually indications, rather than absolute criteria, of the open loop frequency response that will lead to satisfactory closed-loop performance. Therefore, the design objective can be seen as to find a compensator $C(s)$ of lowest order, such that the loop gain frequency response stays in a close neighborhood of the points specified in Eq. (2.3). The tolerance of error can be predetermined by the designer and a search algorithm can be used to find the solution. This algorithm will repeatedly solve Eq. (2.5) while increasing the order of $n(s)$ and $d(s)$ until it finds the solution within the error tolerance. 
2.2.3. Equality via inequality constraints. The loop gain constraints in the loopshaping design approach are mostly given as a set of inequalities, such as $|L(j \omega)|>\alpha$, for $\omega_{1}<\omega<\omega_{2}$, etc. This is obviously more flexible than the equality constraints shown in Eq. (2.1). In determining the compensator, however, the equality constraints are easier to use in order to solve for the coefficients of $C(s)$ algorithmically. Note that the solutions of Eq. (2.5) are usually least-square solutions and they do not solve Eqs (2.1)-(2.3) exactly. This is acceptable since the design specification, and therefore the loop gain constraints, are not absolute in nature. The constraints must be selected reasonably so that they can be met by using a relatively simple compensator. For example, if the loop gain is required to have the magnitude decreasing over a frequency range, one should allow the phase to drop over the same range.

2.2.4. Modeling of the plant. A unique feature of this design approach is that it does not require the explicit mathematic model of the plant. To carry out the design, the only information needed from the plant is its frequency response as a set of frequencies, $\left\{\omega_{i}\right\}$. Consequently, not only the major portion of the system identification process is eliminated, but also the errors associated with it. Furthermore, it makes it feasible to implement an automatic design process on-line such that the compensator can be adjusted as the dynamics of the plant changes. This will be addressed in detail later in Section IV.

2.2.5. Control reconfiguration. Control system redesign is often necessary in many applications, such as failure accommodations (11), where the plant dynamics change significantly during operation. Such tasks can be carried out with the proposed approach. Unlike many existing techniques, there is no need to estimate the system parameters on-line since only the frequency response is required by the tuning algorithm. Therefore, it makes the practical implementation of such systems more realistic. Furthermore, the new design method offers a unique way to stabilize the impaired system quickly by solving a set of linear algebraic equations. Constraints for both stability and performance are expressed in the same equations and the compensator that meets these constraints is obtained in one step. The degree of the compensator is not predetermined and this offers great flexibility in accommodating a wide range of failures.

\section{Example 1}

Consider the feedback control system shown in Fig. 1. Assume that $g(s)=1 /(s+1)(s+5)$ and the design specifications are as follows: the crossover frequency be around $\omega=1 \mathrm{rad} / \mathrm{s}$; the output disturbance be attenuated at least 40 $\mathrm{dB}$ for $\omega \leqslant 0.01 \mathrm{rad} / \mathrm{s}$; the gain and phase margin be above 4 and $30^{\circ}$, respectively; and finally, the system remains stable when there is unmodeled dynamics of the magnitude up to $40 \mathrm{~dB}$ for $\omega \geqslant 10 \mathrm{rad} / \mathrm{s}$.

Translating the closed-loop specifications to loop constraints, the crossover frequency and stability margin conditions are directly applied to the loop gain; the disturbance rejection and stability robustness conditions can be interpreted as $|P(j \omega) C(j \omega)| \geqslant 100, \omega \leqslant 0.01 \mathrm{rad} / \mathrm{s}$, and $|P(j \omega) C(j \omega)|<0.01, \omega \geqslant 10 \mathrm{rad} / \mathrm{s}$. From these constraints, four interpolation pairs are selected as shown in Table $I$. 
TABLE I.

Interpolation constraints

\begin{tabular}{lcccc}
\hline$\omega(\mathrm{rad} / \mathrm{s})$ & 0.01 & 1 & 2 & 10 \\
\hline$|P(j \omega) C(j \omega)|$ & 100 & 1 & 0.2 & 0.005 \\
$\arg (P(j \omega) C(j \omega))$ & $-90^{\circ}$ & $-130^{\circ}$ & $-160^{\circ}$ & $-270^{\circ}$ \\
$|C(j \omega)|$ & 500 & 10 & 2.5 & 0.67 \\
$\arg (C(j \omega))$ & $-84^{\circ}$ & $-70^{\circ}$ & $75^{\circ}$ & $-120^{\circ}$ \\
\hline
\end{tabular}

Note that the interpolation constraints are selected with some conservatism so that the inaccuracies in the approximate solutions can be tolerated to a certain degree.

Now, the solutions are obtained by solving Eqs (2.1)-(2.8). All solutions with order higher than one solve the least-square problem with a negligible error. An approximate first-order solution with the error in a reasonable range is found to be:

$$
C(s)=\frac{-0.26 s+6.27}{s} .
$$

The resulting crossover frequency is $0.9 \mathrm{rad} / \mathrm{s}$; gain margin is 3.8 , phase margin is about $35^{\circ} ;|P(j \omega) C(j \omega)|=104 ;$ at $\omega=0.01,|P(j \omega) C(j \omega)|=0.006$ at $\omega=10$ and dropping.

\section{Remark}

There is a trade off between the order of the compensator and how close the interpolation constraints can be met. If one selects the order of the compensator high enough, the constraints in Table I can always be satisfied exactly. The selection of the interpolation pairs also affects the complexity of the solution. Note that, in this example, the specifications on the phase of $L(j \omega)$ are important only around the crossover frequency. The loop gain constraints at low and high frequencies are merely magnitude constraints. However, the choices of the phase at these frequencies can directly influence the structure of the loop gain and the compensator. For example, the constraint of $\arg (P(j \omega) C(j \omega))=-90^{\circ}$ at $\omega=0.01 \mathrm{rad} / \mathrm{s} \mathrm{implies}$ that the loop gain will have one pole at the origin.

\section{A Novel Self-tuning Control Scheme}

Under various circumstances, the dynamics of the physical process will change. The change may happen quickly or slowly depending on the nature of the plant. For example, the performance of actuators may degrade slowly with time which corresponds to slow changes in the dynamics of the system. On the other hand, if a failure suddenly occurs in an actuator, it will introduce dramatic variations in the system which corresponds to quick changes in system dynamics. In either situation, the compensator $C(s)$ designed for the original plant $P(s)$ may become 


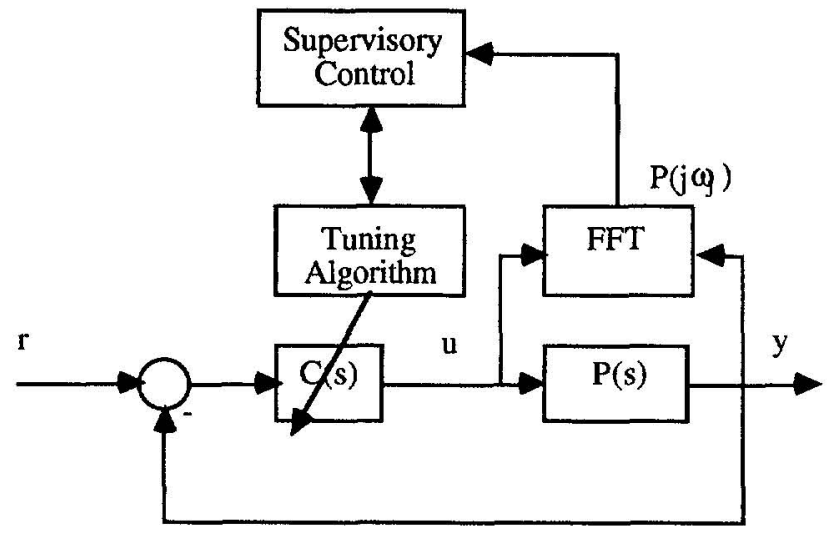

FiG. 2. A self-tuning control system.

ineffective and need to be adjusted during operation. In the following, a self-tuning control system is proposed to address such problems.

The new design approach discussed above integrates the modeling and design into one process. Once the design specifications are given in terms of loop gain frequency response, the rest of the design can be carried out by a computer algorithm. The frequency response of the plant can be found as the ratio of Fourier transform of the input and output. Or, it can be calculated as (12):

$$
P\left(j \omega_{i}\right)=\frac{S_{u u}\left(j \omega_{i}\right)}{S_{u y}\left(j \omega_{i}\right)},
$$

where $S_{u t}\left(j \omega_{i}\right)$ and $S_{u y}\left(j \omega_{i}\right)$ are the auto- and cross spectra of the input and output time history. With $L\left(j \omega_{i}\right)$ and $P\left(j \omega_{i}\right)$ given for $i=1, l$, the compensator $C(s)$ is obtained by solving the linear algebraic equation (2.5) on-line.

\subsection{System configuration}

Based on the above discussion, a conceptual configuration of a self-tuning control system is shown in Fig. 2. In this system, the input and output data in time domain is continuously recorded and the frequency response $P\left(j \omega_{i}\right)$ is obtained using the fast Fourier transform (FFT). From the new $\left\{\boldsymbol{P}\left(j \omega_{i}\right)\right\}$ and the given constraints on loop gain, $\left\{L\left(j \omega_{i}\right)\right\}$, the supervisory control, a higher level decisionmaking mechanism, determines of the compensator should be updated. This is done by comparing the frequency response of the loop gain transfer function at a set of frequencies $\left\{\omega_{i}\right\}$ with the desired one. If the difference exceeds a predetermined limit, the tuning algorithm will be executed. The tuning algorithm receives $P\left(j \omega_{i}\right)$ and $L\left(j \omega_{i}\right)$ from the supervisory control block and determines the new compensator $C(s)$ using the design method discussed in Section II. Thus, as the dynamics of the plant changes, the performance of the closed-loop system is maintained by adjusting $C(s)$. This process can be completely automated without human intervention. Furthermore, the computational complexity of the algorithm is expected to be reasonable for on-line operation since it only involves solving a set of linear algebraic equations. 


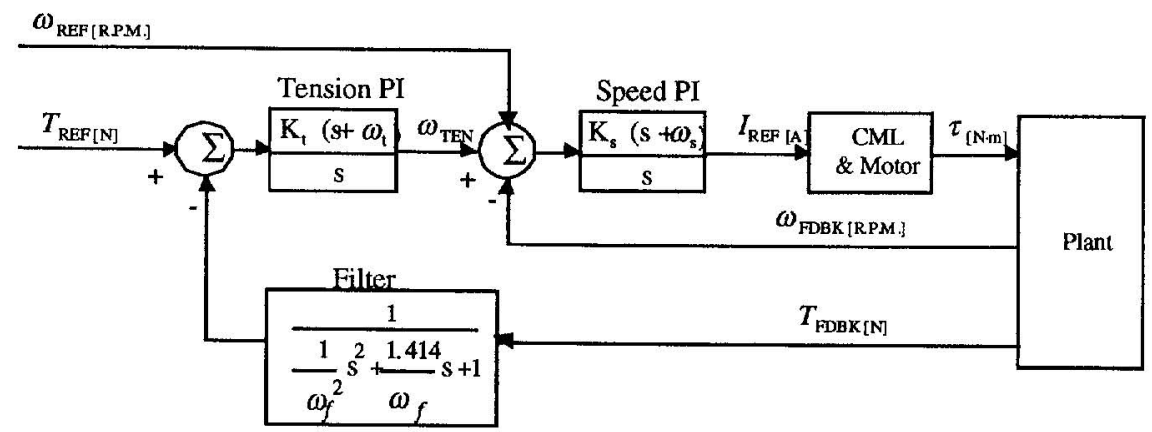

FIG. 3. A typical web tension regulation scheme.

For relatively slow changes in the plant dynamics where the system is still approximately linear and time invariant, the adjustment of the compensator will be infrequent. For dramatic changes in the system such as component failures or surface damages in aircraft, it is essential that the system be quickly stabilized before it gets out of control. The tuning method proposed here could be very effective in achieving this goal since it does not require the explicit model of the plant and a control redesign can be quickly executed. For systems that are essentially nonlinear but can be approximated by a linear model in a certain range of frequencies, the frequency response of the plant, $P\left(j \omega_{i}\right)$, indirectly approximates the nonlinear system with a linear system that has the transfer function $P(s)$. Thus, the explicit modeling and linearization of nonlinear systems are not necessary.

\subsection{Comparison to self-tuning adaptive control}

The proposed method is similar in concept to self-tuning adaptive control. The objective of both methods is to adjust the compensator to accommodate the changes in the plant. The implementations are, however, very different. The new method has the following unique characteristics:

(a) It does not estimate the parameters of the plant, directly or indirectly. Therefore, there are no assumptions made regarding the structure, the order, the relative degree, etc., of the plant.

(b) There is no assumption made on the structure of the compensator. The order of the compensator is determined only to satisfy the design constraints. Compromises can be made automatically between the complexity of $C(s)$ and the performance specifications.

(c) The compensator is only adjusted when necessary and it is done quickly in one step. For this purpose, a decision-making mechanism, perhaps in the form of a rule-based system, is required.

\section{Example 2. Self-tuning web tension regulation}

The industrial WEB tension regulators are typically implemented with a PI controller as shown in Fig. 3. The physical plant is shown in Fig. 4, where the unstretched web is introduced into the plant. The PI parameters are tuned to 


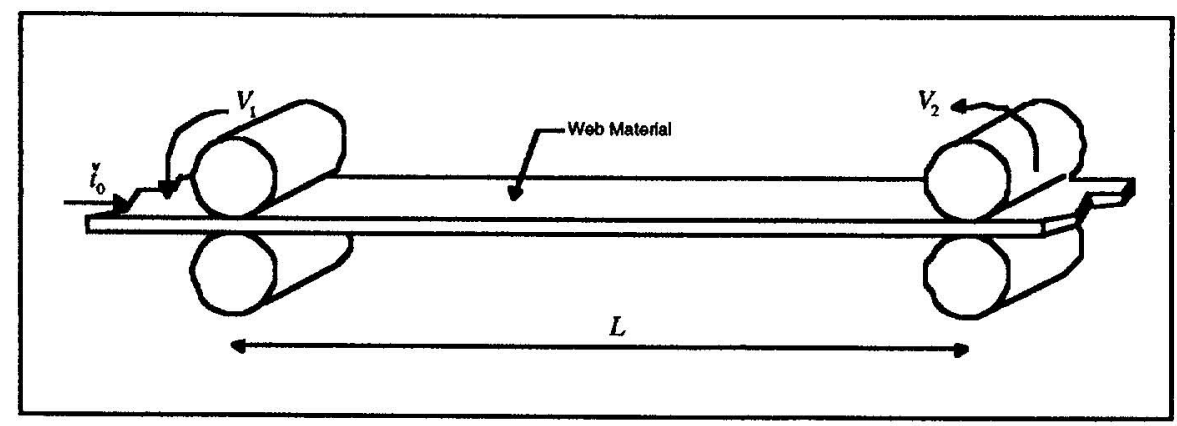

FIG. 4. The physical plant.

provide a stable responsive system for the entire range of product processed through the system. This results in a system that is de-tuned for a large range of products and optimally tuned for a small range of products.

Unknowns such as web damping, friction and slippage make the derivation of analytical tuning algorithms difficult. The usual approach is to tune heuristically the PI controller on-site based on observed system performance. Typically this results in stable tension regulation until such time as a product with extreme physical parameters is processed through the system. All too often this results in unstable tension regulation which in turn requires re-tuning of the loop. A better approach would be to provide a self-tuning regulation scheme that obviates the need for empirically finding a single unique set of stable tuning parameters for the entire range of products processed through the system.

To this point adaptive and/or self-tuning regulation schemes have not been considered for web transport systems. This fact can be attributed to the complexity of the existing time-domain algorithms, and the associated hardware and software implementation difficulties.

The use of a PI controller for the inner speed loop is desirable for actual implementations where the need to jog the driven roll without the web material connected is a necessity. Assuming: (1) the tension in the web entering the tension zone is zero and (2) the system speed reference $V_{1}$ is constant, the following transfer function from the output of the tension regulator to the tension feedback can be derived:

$$
\frac{T_{\mathrm{FDBK}}}{\omega_{\mathrm{TEN}}}=\frac{\frac{K L}{V_{1} K_{2}}\left(\frac{s}{\omega_{s}}+1\right)}{\frac{J L}{K_{s} V_{1} \omega_{s}} s^{3}+\frac{J V_{1}+K_{s} L}{K_{s} V_{1} \omega_{s}} s^{2}+\frac{K L+K_{s}\left(L \omega_{s}+V_{1}\right)}{K_{s} V_{1} \omega_{s}} s+1},
$$

where $E=$ web modulus of elasticity $\left(\mathrm{N} / \mathrm{cm}^{2}\right), A=$ web cross-sectional area $\left(\mathrm{cm}^{2}\right)$, $L=$ web length $(\mathrm{m}), V_{1}=$ velocity of feed roll $(\mathrm{m} / \mathrm{s}), V_{2}=$ velocity of exit roll $(\mathrm{m} / \mathrm{s}), \omega_{s}=20(\mathrm{rad} / \mathrm{s}), J$ is lumped roll/motor inertia $\left(\mathrm{N} \mathrm{m}^{2}\right)$ and $K=K_{1} K_{2}(E A / L)$.

The self-tuning web tension regulator was implemented as shown in Fig. 2. The simulation was performed using SIMULINK and MATLAB. Viscous friction, 

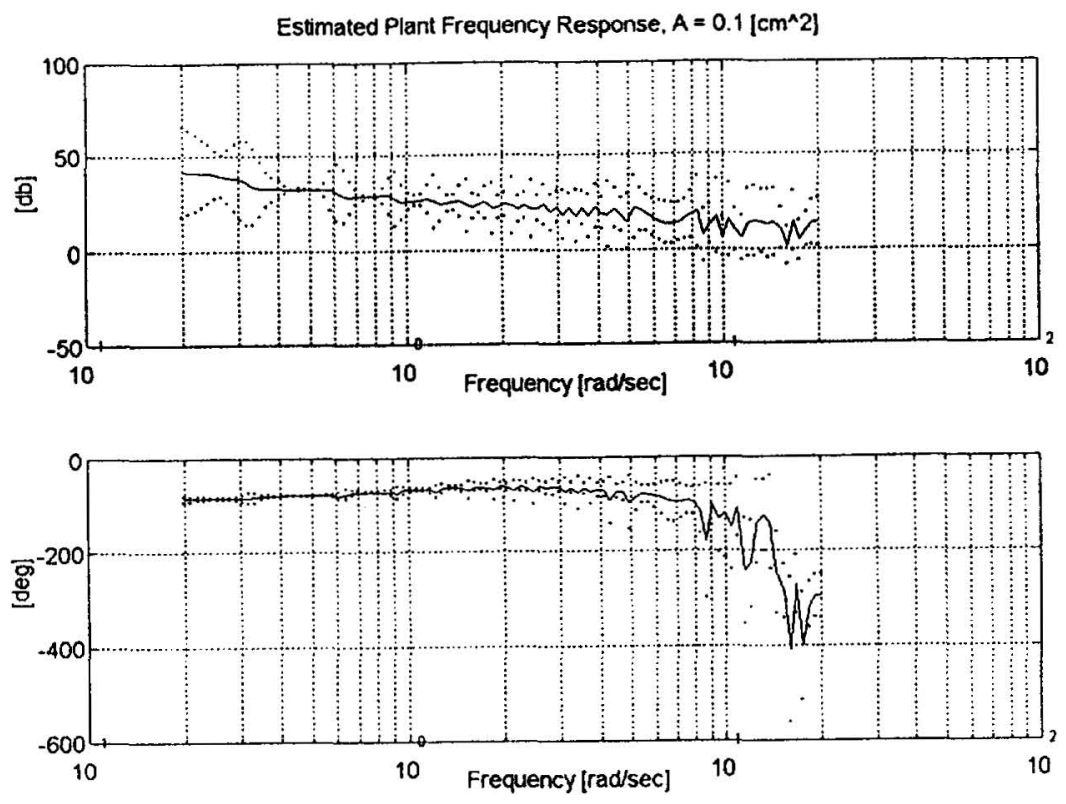

FIG. 5. Output response before and after self-tuning $\left(A=10 \mathrm{~cm}^{2}\right)$.

feedback noise, line speed noise and web-damping were included to provide realistic unmodeled dynamics. A SIMULINK masked $s$-function performs the FFT and executes the self-tuning algorithm to provide the new controller parameters. During the self-tuning process the system is stimulated with a small speed reference step. Care was taken to ensure that web tension was reasonably bounded during this period. To prevent regulator wind-up while the system is run with an open tension loop during the plant identification process, the input to the tension regulator is forced to zero.

The self-tuning system was initialized with a set of poorly tuned controller parameters. Two tension steps of 5-s duration (from 0 to $10 \mathrm{~s}$ in Fig. 6) were followed by a 10 -s data collection and regulator tuning period. After which the retuned response to three tension steps of 5-s duration was obtained. The plant frequency response obtained via the FFT is shown in Fig. 5 with probabilistic bounds of error.

Note that the ability of the self-tuning algorithm to tune the regulator such that the desired open loop frequency response is obtained is directly related to the ability of the application FFT algorithm to identify the frequency response of the plant. It was observed that the relatively accurate plant frequency response data, as shown in Fig. 5, is critical to the success of the application of the described selftuning algorithm. Factors impacting the quality of the estimated plant frequency response are: (1) sample time (high frequency response), (2) the sample length (low frequency response), (3) the choice of FFT windowing algorithm (transient disturbance and noise rejection), (4) the choice of smoothing algorithm (noise rejection) and (5) harmonic content of the input stimulus (overall frequency 


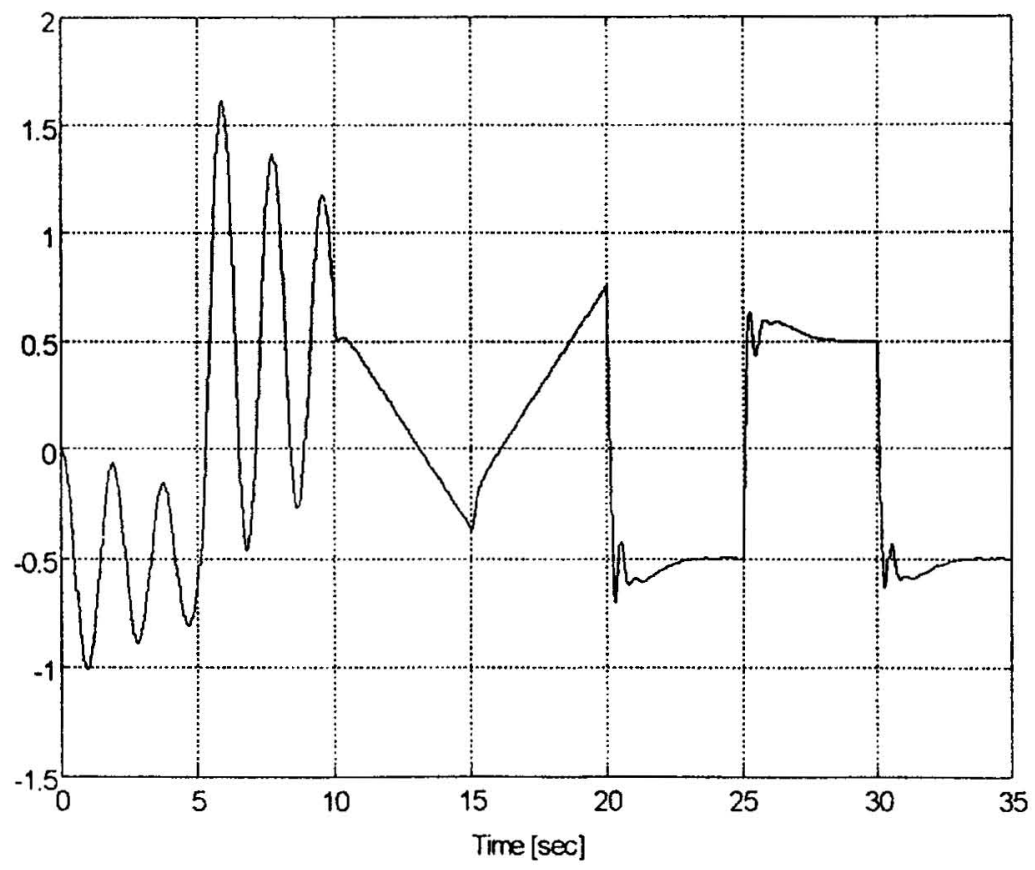

FIG. 6. Estimated frequency response of the plant $\left(A=10 \mathrm{~cm}^{2}\right)$.

response). Adequate performance of the tuning will depend on making appropriate choices of (1) to (5) based on the given application.

Simulation results also indicate that the choice of frequencies used in the generation of $L(j \omega)$ and $P(j \omega)$ govern the quality of the final self-tuned regulator design. Care should be taken to provide frequencies low enough to generate a meaningful estimation of the plant's low-frequency response yet high enough to provide for adequate compensation of high-frequency underdamped modes.

\section{Extension to Discrete Time Systems and MIMO Systems}

\subsection{Discrete time system design}

The new design method described above can be directly extended to discrete time control systems. Assuming that the plant is a discrete time system with a transfer function $P(z)$, the compensator is $C(z)$, and the loop gain transfer function is $L(z)=P(z) C(z)$. The frequency response of discrete time systems is obtained by substituting $z$ as $z=\mathrm{e}^{j \omega T}$, where $T$ is the sampling period. The curve-fitting problem in discrete time (7) can be described as given the input and output data in frequency domain, i.e. $u\left(\mathrm{e}^{j \omega T}\right)$ and $y\left(\mathrm{e}^{j \omega T}\right)$ at a set of discrete frequencies $\left\{\omega=\omega_{i}, i=1,2, \ldots\right\}$, respectively, find a transfer function matrix $H(z)$ such that:

$$
H\left(\mathrm{e}^{j \omega_{i} T}\right) u\left(\mathrm{e}^{j \omega_{i} T}\right)=y\left(\mathrm{e}^{j \omega_{i} T}\right), \quad i=1,2, \ldots
$$

Let the design specifications be expressed as interpolation constraints of the form: 


$$
L\left(\mathrm{e}^{j \omega_{i} T}\right)=\alpha_{i}, \quad i=1, l,
$$

where $x_{i}$ are complex numbers. Similar to the continuous time system, the design problem becomes to find a compensator $C(z)$ such that:

$$
L\left(\mathrm{e}^{j \omega_{i} T}\right)=P\left(\mathrm{e}^{j \omega_{t} T}\right) C\left(\mathrm{e}^{j \omega_{i} T}\right), \quad i=1, l,
$$

where $P\left(\mathrm{e}^{\mu \omega_{i} T}\right)$ is the given frequency response of the plant evaluated at $\omega_{i}$. Writing $L\left(\mathrm{e}^{j \omega_{i} T}\right)=\alpha_{i}$ and $P\left(\mathrm{e}^{j \omega_{i} T}\right)=\beta_{i}, i=1, l$, the numerator and denominator coefficients of $C(z)$ can be obtained by solving the set of linear algebraic equations:

$$
C\left(\mathrm{e}^{j \omega_{i} T}\right) \beta_{i}=\alpha_{i}, \quad i=1, l .
$$

Given the degree of $C(z), \alpha_{i}$ and $\beta_{i}$, the problem of determining the transfer function $C(z)$ that satisfies the constraints in Eq. (4.4) can be solved by using matrix interpolation theory as well (7). This is shown as follows.

Let $C(z)$ be $C(z)=n(z) / d(z)$, where $n(z)$ and $d(z)$ are numerator and denominator polynomials, respectively. The problem in Eq. (4.4) becomes determining the polynomial matrix $[n(z),-d(z)]$ which satisfies

$$
\left[n\left(\mathrm{e}^{j \omega_{i} T}\right),-d\left(\mathrm{e}^{i \omega_{i} T}\right)\right]\left[\begin{array}{c}
\beta_{i} \\
\alpha_{i}
\end{array}\right]=0, \quad i=1, l,
$$

where $l$ is the number of constraints.

Given column degrees of $n(z)$ and $d(z), d_{1}$ and $d_{2}$, and $l$ constraints $\left\{\mathrm{e}^{j \omega_{i} T}, \beta_{j}, \alpha_{j}\right\}$, the matrix $[n(z),-d(z)]$ can be determined from

$$
[N,-D]\left[S_{l}, C\right]=[0, E],
$$

where

$$
[n(z),-d(z)]=[N,-D] S(z)
$$

with

$$
\begin{gathered}
S(z)=\operatorname{blk} \operatorname{diag}\left\{\left[1, z, \ldots, z^{d_{d}}\right]^{\prime}\right\}, \quad i=1,2 \\
S_{l}:=\left[S\left(\mathrm{e}^{j \omega_{1} T}\right) c_{1}, \ldots, S\left(\mathrm{e}^{j \omega_{l} T}\right) c_{l}\right] .
\end{gathered}
$$

Here $N$ and $D$ are row vectors which contain the coefficients of $n(z)$ and $d(z)$, respectively; $c_{i}=\left[\beta_{i}, \alpha_{i}\right]^{\prime}$. Similar to the continuous time curve-fitting problem, $C$ and $E$ are used to impose extra constraints on $n(s)$ and $d(s)$.

Note that the frequency response of a discrete time system is periodic. In loop shaping, one only needs to consider the frequency response in the first period. That is, the frequencies of interest are limited to a small range and the numerical properties of Eq. (4.6) are usually better than those of Eq. (2.5).

\subsection{MIMO system design}

The concept of the classical frequency domain design methods for SISO systems have already been extended to MIMO systems $(2,3)$. Consider the system in Fig. 1 where $P(s)$ and $C(s)$ are now $q \times p$ and $p \times m$ transfer function matrices, 
respectively. The same design philosophy can be applied using the singular value plot of $L(j \omega)=P(j \omega) C(j \omega)$, instead of the Bode plot. Similarly, the design specifications can be expressed in terms of the singular value plot of $L(j \omega)$ and the compensator $C(s)$ is to be found so that $L(j \omega)$ meets the constraints. Here the problem is finding $C(s)$ is rather difficult due to the lack of intuition on the relation between $C(s)$ and the singular value plot of the loop gain. Even if a solution is found, the design is likely to be very conservative since the singular value of a matrix is only related to the bound on the absolute value of its eigenvalues. That is, only the information on the upper and lower bounds of the magniture of the transfer function matrix is used in design.

The extension of the new design method to MIMO system design requires the design specifications be expressed in terms of frequency response of the loop gain transfer function $L(s)$ at a set of discrete frequencies, $L\left(j \omega_{i}\right), i=1, l$. Once this is accomplished, the same procedure for SISO systems can be applied with few modifications. This is briefly described below.

Assume that the left coprime fraction representation of $C(s)$ is

$$
C(s)=\tilde{D^{-1}}(s) \tilde{N}(s),
$$

where $\tilde{D}(s)$ and $\tilde{N}(s)$ are $(p \times p)$ and $(p \times m)$ polynomial matrices, respectively; the design specifications are given in the same way as in Eqs (2.1)-(2.3), except $\alpha_{i}$ and $\beta_{i}$ are now vectors of appropriate dimensions. The problem becomes determining the polynomial matrix $[\tilde{N}(s),-\tilde{D}(s)](p \times(p+m))$ which satisfies

$$
\left[\tilde{N}\left(j \omega_{i}\right),-\tilde{D}\left(j \omega_{i}\right)\right]\left[\begin{array}{c}
\beta_{i} \\
\alpha_{i}
\end{array}\right]=0, \quad i=1, l,
$$

where $l$ is the number of constraints.

Given column degrees of $[\tilde{N}(s),-\tilde{D}(s)], d_{i}, i=1, p+m$, and $l$ constraints $\left\{j \omega_{i}\right.$, $\left.u\left(\omega_{i}\right), y\left(\omega_{i}\right)\right\}$, based on rational matrix interpolation theory $(5)$, the matrix $[\tilde{N}(s)$, $-\tilde{D}(s)]$ can be determined from

$$
[\tilde{N},-\tilde{D}]\left[S_{l}, C\right]=[0, E],
$$

where

$$
[\tilde{N}(s),-\tilde{D}(s)]=[\tilde{N},-\tilde{D}] S(s)
$$

with

$$
\begin{gathered}
S(s)=\operatorname{blk} \operatorname{diag}\left\{\left[1, s, \ldots, s^{d_{1}}\right]^{\prime}\right\}, \quad i=1, p+m \\
S_{l}:=\left[S\left(j \omega_{1}\right) c_{1}, \ldots, S\left(j \omega_{l}\right) c_{l}\right],
\end{gathered}
$$

where $c_{i}=\left[\beta_{i}, \alpha_{i}\right]^{\prime}$.

\section{Remark}

It is shown here that the new loop-shaping design technique can be directly applied to MIMO systems. A unique challenge in MIMO system design is to translate all design specifications as loop gain constraints. In applications such as 
failure accommodation and self-tuning control, this can be achieved by using the information from the nominal (or desired) system model. Further investigation is needed to deal with the issue in a more general setting.

\section{Concluding Remarks}

It is shown in this paper that the proposed loop-shaping design technique has a great potential to relieve control design engineers from the tedious task of manipulating the loop gain frequency response in order to achieve design specifications. This innovative design approach also leads to a novel self-tuning scheme which was successfully implemented and simulated for an industrial application.

\section{Acknowledgement}

The author would like to thank Mr Brian Boulter from Reliance Electric Corporation for providing the background, the system model and data, and computer simulation in Example 2.

\section{References}

(1) C. E. Rohrs, J. L. Melsa and D. G. Schultz, "Linear Control Systems", McGraw-Hill, New York, 1993.

(2) J. M. Maciejowski, "Multivariable Feedback Design". Addison Wesley, New York, 1989.

(3) J. C. Doyle, B. A. Francis and A. R. Tannenbaum, "Feedback Control Theory". Macmillan, New York, 1992.

(4) K. J. Astrom, C. C. Hang, P. Persson and W. K. Ho, "Towards intelligent PID control", Automatica, Vol. 28, pp. 1-9, 1992.

(5) P. J. Antsaklis and Z. Gao, "Polynomial and rational matrix interpolation: theory and control applications", J. Control Int., Vol. 58, pp. 349-404, 1993.

(6) E. C. Levy, "Complex curve fitting", IEEE Trans. Autom. Control, Vol. AC-4, 1959.

(7) Z. Gao, B. Tabachnik and R. Savescu, "A new approach to multi-input and multioutput system identication in frequency domain", J. Franklin Inst. Vol. 331 B, pp. 435-448, 1994.

(8) R. V. Savescu, "System identification from frequency response using matrix interpolation theory", M.S. Thesis, Dept. of Electrical Engineering, Cleveland State University, 1992.

(9) B. Tabachnik, "A computer algorithm for multi-input multi-output system identication using Chebychev polynomials", M.S. Thesis, Dept. of Electrical Engineering, Cleveland State University, 1994.

(10) C. L. Lawson and R. J. Hanson, "Solving Least Squares Problems", Prentice-Hall, Englewood Cliffs, NJ, 1974.

(11) Z. Gao, "Techniques in reconfigurable control system design", in "Digital Design and Control Systems Techniques and Applications" (Edited by C. T. Leondes), Academic Press, New York, 1995 (to appear).

(12) J. S. Bendat and A. G. Piersol, "Engineering Applications of Correlation and Spectral Analysis", Wiley, New York, 1980. 